\title{
Impact of host factors on susceptibility to antifungal agents
}

\author{
Balbina Plotkin $^{1 *}$, Monika Konaklieva ${ }^{2}$ \\ ${ }^{1}$ Department of Microbiology and Immunology, College of Graduate Studies, Midwestern University, Downers Grove, IL \\ 60515 \\ ${ }^{2}$ Department of Chemistry, American University, College of Arts and Sciences, 4400 Massachusetts Ave, NW, \\ Washington, DC 20016
}

*Corresponding Author: E-mail: bplotk@midwestern.edu; Tel.: +1-630-515-6163; Fax: +1-630-515-7249

Received: November 08, 2021; Accepted: December 30, 2021; Published: January 07, 2022

\begin{abstract}
An obstacle to drug development, particularly in this era of multiple drug resistance, is the underappreciation for the role the host environment plays in microbial response to drugs. With the rise in fungal infection and drug resistance, particularly in individuals with co-morbidities, the influence serum and its components have on antimicrobial susceptibility requires assessment. This study examined the impact of physiologically relevant glucose and insulin levels in the presence and absence of $50 \%$ human plasma on MICs for clinical isolates of Candida lusitaniae, Candida parapsilosis, Candida albicans, Candida tropicalis, Candida glabrata, Candida krusei and Cryptococcus neoformans. The addition of insulin or glucose at physiologic levels in RPMI medium alone altered the MIC in either a positive or negative fashion, depending on the organisms and drug tested, with C. glabrata most significantly altered with a 40, >32and 46-fold increase in MIC for amphotericin B, itraconazole and miconazole, respectively. The addition of candida-antibody negative plasma also affected MIC, with the addition of glucose and insulin having a tandem effect on MIC. These findings indicate that phenotypic resistance of Candida and Cryptococcus can vary depending on the presence of insulin with glucose and plasma. This modulation of resistance may help explain treatment failures in the diabetic population and facilitate the development of stable drugresistant strains. Furthermore, these findings indicate the need for a precision approach in the choice of drug treatment and drug development.
\end{abstract}

(C)2022 by the authors. This article is an open-access article distributed under the terms and conditions of the Creative Commons Attribution license (http://creativecommons.org/licenses/by/4.0/).

\section{Keywords}

Insulin; glucose; human serum; Candida; Cryptococcus.

\section{Introduction}

Phenotypic response to environmental shifts is essential for microbial survival and is of particular importance with respect to changes that occur in vivo, which affect response to antimicrobial agents. The potential changes in response to drugs upon growth within the host could both be responsible for treatment failures and provide the potential for novel drug development. Thus, it is essential to define these phenotypic responses to host factors, and metabolic conditions, to predict effective drug design and utility.

A common metabolic condition associated with increased risks for infections is type II diabetes. People 
with type II diabetes can exhibit hyperglycemia, hyperinsulinemia, and/or combined hyperglycemiahyperinsulinemia, as a result of insulin intolerance [1,2]. A metabolic immunosuppressive condition, type II diabetes, places individuals at increased risk for fungal diseases [3-6]. Thus, during the infectious process, a pathogen can be exposed to elevated levels of insulin and/or glucose as compared to the metabolically normal host.

A critical disconnect regarding predicted sensitivity via in vitro testing and efficacy is the lack of congruency with the metabolic disorders most commonly at risk for fungal infections, e.g., type II diabetes, since antimicrobial activity testing is predicated on the host being in a normal metabolic state. Most studies examining serum effects are focused on the impact normal serum/plasma has on antimicrobial activity, or on the effect of high glucose $(2 \mathrm{mg} / \mathrm{dl})$ concentrations, which are reported to affect the ability of antifungal agents to bind to their targets [7-9]. To date, studies of glucose-fungal-antifungal agent interactions fail to take into consideration the concomitant presence of insulin with glucose. This becomes important since organisms across the taxonomic kingdoms are reported to produce and/or react to insulin [10-16]. In Candida albicans, insulin has been associated with increased expression of virulence-associated morphological transition, from blastospore to hyphal production [17]. The mechanism via which insulin promotes yeast to hyphal transition is by enhancing uptake of proline by $C$. albicans. Conversely, glucose concentrations can affect morphogenic expression $[18,19]$. In the present study, the effect on in vitro antifungal activity in the presence and absence of human plasma by insulin and/or glucose, at levels analogous to those reported in type II diabetes, was determined.

\section{Experimental}

\section{Fungal species and culture}

Candida lusitaniae, Candida parapsilosis, Candida albicans, Candida tropicalis, Cryptococcus neoformans, Candida glabrata, and Candida krusei clinical isolates (a generous gift from the lab of Paul Schreckenberger, Loyola University Medical School) were maintained at $-70^{\circ} \mathrm{C}$ and passed at least twice on Sabouraud dextrose agar (SDA) prior to testing.

\section{Drug testing}

Antifungal activity determinations were done using the standard CLSI (NCCLS M27-T) microbroth dilution method with modifications [20,21]. Susceptibility to amphotericin B (Sigma-Aldrich), fluconazole (Pfizer), itraconazole (Janssen Pharmaceuticals), and miconazole (Sigma-Aldrich) was determined using antifungal agents constituted and serially diluted. Microtiter plates containing the serial dilutions of the antifungal agents were made as recommended and stored frozen $\left(-70^{\circ} \mathrm{C} ; \leq 7\right.$ days) or refrigerated (itraconazole; $\leq 3$ days). Antifungal activity in the presence or absence of normal human plasma (type $A, R h$ + ), was performed using $2 X$ RPMI medium ( $50 \% \mathrm{v} / \mathrm{v}$ ). Plasma was screened for specific fungal antibodies, as described below, by indirect immunofluorescent microscopy (FITC-Protein A) to detect the presence of yeast-bound antibodies. Minimum lethal concentrations (MLC) were determined by plating $10 \mu \mathrm{l}$ of all wells showing no growth onto SDA.

To simulate glucose and insulin levels reported for type II diabetes, glucose $(285 \mathrm{mg} / \mathrm{dl})$ and/or insulin $(200 \mu \mathrm{U} / \mathrm{ml})$, final concentrations were tested in RPMI with and without $50 \%$ plasma medium. Endogenous glucose levels were determined using a kit according to the manufacturer's instructions (Sigma-Aldrich). The reported mid-range value for normal human insulin levels $(18 \mu \mathrm{U} / \mathrm{ml})$ was used as the basis for calculating the amount of insulin to add. Glucose and/or insulin in RPMI medium had no effect (data not 
shown) on fungal growth kinetics as determined by both turbidimetric measurements (absorbance 560 $\mathrm{nm}$ ) and a direct microscopic count of yeast and hyphal forms. The growth rates and the relative production of yeast and hyphal forms during growth in RPMI medium with and without glucose and/or insulin were measured to determine if changes in the MIC and MLC of the antifungal agents tested in the presence of glucose and/or insulin could be correlated to alterations in fungal growth. No significant differences in generation time or proportion of yeast and hyphal forms were measured. The differences in the rate of growth between organisms also appear to be unrelated to the alterations in MIC and MLC measured in the presence of glucose and/or insulin and to the relative susceptibility of the organisms. The antifungal agents were not exposed to the media supplements (glucose and insulin) until organisms were added to the agent. Sterility controls and growth controls for all test conditions were run simultaneously with the tests.

To determine antifungal activity in the presence of normal human plasma (type $A, R h+$ ), a modified protocol was developed. Plasma was screened for specific fungal antibodies by indirect immunofluorescent microscopy using FITC-Protein A to detect the presence of bound antibodies. Equal volumes of normal human plasma were added to 2 X NCCLS RPMI medium to yield a medium that contained $1 \mathrm{X}$ RPMI with 50 $\% \mathrm{v} / \mathrm{v}$ of plasma. For glucose in a medium containing $50 \%$ plasma (plasma medium), glucose and/or insulin were added to ensure equivalent amounts were used for the medium alone. Antifungal agents were prepared, as described above, in this plasma medium. Antifungal activity in the presence and absence of plasma was determined on the same day using the same drug lot and microbial suspension. Minimum inhibitory concentrations (MIC) were determined by visual inspection of the wells at 24, 36 and $72 \mathrm{hrs}$ after initiation of the incubation period. Minimum lethal concentrations (MLC) were determined by plating $10 \mu \mathrm{l}$ of all wells showing no growth onto Sabouraud dextrose agar (Difco) at 36 hrs post-initiation of the incubation period.

\section{Results and discussion}

\section{Amphotericin B}

The overall effect of insulin and glucose alone or together were drug and fungal species-specific (Table 1). Amphotericin $B(A m B)$ activity was relatively unaffected by insulin and glucose combined with the exception of $C$. glabrata (40-fold greater MIC than RPMI alone; Table 1). For AmB, the presence of plasma with and without glucose and/or insulin decreased the $\mathrm{MIC}<4$ fold of that measured for medium alone for all organisms, with the exception of $C$. glabrata and C. neoformans. For amphotericin B in plasma supplemented with glucose, its MIC increased from four to a maximum of 32 -fold for $C$. krusei compared to medium with plasma alone (Table 2). Like with itraconazole, with amphotericin B, there was a difference between the MIC and MLC. The MLC of $C$. albicans was 32-fold higher than the MIC in the presence of plasma without supplements. The plasma effect on the candidal MLC was overcome by the addition of glucose and/or insulin. The MLC was within 2-fold of the amphotericin B MIC for all other isolates. Comparison of medium with plasma to medium alone (Table 3 ) indicated that the addition of $50 \%$ normal human plasma to medium decreased the MIC of amphotericin B > 16-fold for all isolates, except C. lusitaniae and C. krusei, which were unaffected.

\section{5-Fluorocytosine}

The MIC of 5 -fluorocytosine in media supplemented with glucose alone either was unaffected ( $C$. lusitaniae, C. neoformans, and C. krusei), or decreased (Table 1). The effect of insulin on the MIC in media was also isolate-dependent. Insulin alone had the greatest negative impact on 5-fluorocytosine activity for C. tropicalis ( 25 -fold), with C. parasilosis and C. glabrata exhibiting a 6-fold increase in MIC. In general, 
the MIC of all isolates in medium supplemented with glucose and insulin was decreased compared to medium alone, although the degree of decrease was isolate-dependent. The presence of plasma $(50 \% \mathrm{v} / \mathrm{v}$ RPMI medium) resulted in an increase in 5-fluorocytosine MIC maximally for $C$. parasilosis, (32-fold) and $C$. tropicalis (16-fold), as compared to RPMI medium alone for all isolates, with the exception of $C$. glabrata, whose MIC was markedly decreased (Table 2). The MLC in media, and in the presence of plasma with or without supplements correlated within two-fold of the MIC, with the exception of C. glabrata, which in the presence of plasma was four-fold higher than that measured for media alone. The addition of insulin to both medium alone, and with plasma, resulted in an increase in the MIC to a maximum increase measured for C. glabrata when exposed to 5 -fluorocytosine (40-fold increase; $25 \mu \mathrm{g} / \mathrm{ml}$ in media with plasma and insulin vs. $0.625 \mu \mathrm{g} / \mathrm{ml}$ media with insulin alone). Comparison of medium with plasma, to medium alone (Table 3), indicated that the addition of $50 \%$ normal human plasma to medium decreased the MIC 25 -fold for $C$. tropicalis only ( $0.25 \mu \mathrm{g} / \mathrm{ml}$ media with $50 \%$ plasma vs. $6.25 \mu \mathrm{g} / \mathrm{ml}$ RPMI medium alone).

\section{Fluconazole}

Supplementation of RPMI medium containing plasma with insulin, resulted in a 1024-fold increase in MIC of $C$. albicans to fluconazole with the resultant reclassification from sensitive to resistant. However, addition of glucose to insulin restored the sensitivity of $C$. albicans to fluconazole. The only change in classification with regards to fluconazole was a shift from sensitive to sensitive-dose dependent for $C$. lusitaniae when both glucose and insulin were present. In contrast, the presence of plasma resulted in a shift from resistant to sensitive for $C$. neoformans with respect to fluconazole.

\section{Itraconazole}

For itraconazole, the addition of glucose and/or insulin to RPMI medium did not significantly alter the MIC or MLC ( $\leq 2$-fold of MIC in medium alone) for all organisms with the exception of $C$. lusitaniae and $C$. glabrata (Table 1). The addition of glucose and/or insulin to RPMI medium resulted in a MIC for $C$. glabrata that exceeded the drug solubility level (see Table 1 ). The effect of glucose on the $C$. Iusitaniae response to itraconazole was opposite of its effect when combined with insulin (8-fold higher MIC for glucose alone vs. 8-fold lower MIC for glucose and insulin), indicating that there may be an interactive effect of glucose and insulin. The presence of plasma alone had a modest $(<4$-fold) effect on the MIC for all isolates with the exception of $C$. glabrata, where the MIC was decreased nearly 103-fold as compared to that measured for medium alone (Table 2 and 3). The addition of glucose and/or insulin to plasma did not significantly alter the MIC or MLC of itraconazole for all organisms, except for $C$. neoformans, where supplementing plasma with insulin resulted in a 20 -fold decrease in the MIC. Interestingly, the MLC for all conditions tested differed $\leq 2$-fold from the MIC for all organisms, except for C. neoformans (8-fold higher than MIC in plasma supplemented with glucose and insulin) and C. glabrata (128-fold higher than MIC in plasma supplemented with glucose). The MLC for $C$. neoformans in RPMI alone was less affected, exhibiting a fivefold decrease in MLC compared to an MLC measured in plasma alone, or plasma supplemented with glucose. This differential effect of the presence of plasma, as compared to medium alone, may explain the treatment failure reported despite laboratory-confirmed sensitivity [22-27].

\section{Miconazole}

The combination of glucose and insulin resulted in a significant alteration in the miconazole MIC for all isolates except $C$. parapsilosis. Resistance to miconazole was increased by the presence of insulin and glucose to a maximum of 46 -fold and 23-fold over that of RPMI alone for C. glabrata and C. krusei, 
respectively (Table 1 ). The MIC in media supplemented with glucose was decreased from four-fold to a maximum 16-fold measured for $C$. albicans in miconazole. $C$. neoformans was the only organism whose MIC was affected by the presence of insulin. Interestingly, while plasma alone significantly increased drug activity for all drugs tested against $C$. glabrata, the addition of insulin and/or glucose restored this activity to the levels measured for RPMI with plasma alone (Tables 2 and 3). In contrast, the presence of plasma negatively impacted miconazole (C. lusitaniae, > 185-fold; C. tropicalis, > 23-fold; C. krusei, > 92-fold) and with the addition of glucose and insulin, MIC returned to levels within 4-fold that of RPMI with plasma. The addition of glucose to plasma caused either a decrease in the MIC or did not alter the MIC. In all test situations, the MLC correlated with the MIC.

Table 1. Effect of insulin and glucose on fungal MIC $(\mu \mathrm{g} / \mathrm{ml})$

\begin{tabular}{|c|c|c|c|c|c|c|c|c|}
\hline Organism & Drug & RPMI & RPMI + Gluc ${ }^{c}$ & $\begin{array}{c}\text { Ratio } \\
\text { RPMI + Gluc } \\
\text { RPMI }\end{array}$ & $R P M I+\operatorname{Insu}^{d}$ & $\begin{array}{c}\begin{array}{c}\text { Ratio } \\
\text { RPMI + Insu }\end{array} \\
\frac{\text { RPMI }}{}\end{array}$ & $\begin{array}{c}\text { RPMI + } \\
\text { Glucose \& } \\
\text { Insulin }\end{array}$ & $\begin{array}{c}\text { Ratio } \\
\text { RPMI + Gluc \& } \\
\frac{\text { Insu RPMI }}{\underline{M}}\end{array}$ \\
\hline \multirow[t]{5}{*}{ C. Iusitaniae } & $\mathrm{AmB}^{\mathrm{a}}$ & $0.039(\mathrm{~S})^{\mathrm{b}}$ & $0.0195(\mathrm{~S})$ & 0.5 & $0.0195(\mathrm{~S})$ & 0.5 & $0.078(\mathrm{~S})$ & 2 \\
\hline & $5-\mathrm{FC}^{\mathrm{a}}$ & $0.15625(\mathrm{~S})$ & $0.15625(\mathrm{~S})$ & 1 & 0.15625 (S) & 1 & 0.00936 (S) & 0.0599 \\
\hline & Flucon & $4.0(S)$ & $8.0(\mathrm{~S})$ & 2 & $2.0(\mathrm{~S})$ & 0.5 & 16.0 (S-DD) & 4 \\
\hline & $\operatorname{ltra}^{\mathrm{a}}$ & $0.5(S-D D)^{b}$ & $4.0(R)^{b}$ & 8 & 0.5 (S-DD) & 1 & $0.0625(\mathrm{~S})$ & 0.125 \\
\hline & Micon & $0.195(\mathrm{~S})$ & 0.195 (S) & 1 & $0.195(\mathrm{~S})$ & 1 & $2.2425(\mathrm{~S})$ & 11.5 \\
\hline \multirow[t]{5}{*}{ C. parapsilosis } & $\mathrm{AmB}$ & $0.078(\mathrm{~S})$ & 0.0195 (S) & 0.25 & $0.039(\mathrm{~S})$ & 0.5 & $0.078(S)$ & 1 \\
\hline & $5-\mathrm{FC}$ & $0.78(S)$ & $0.39(\mathrm{~S})$ & 0.5 & $4.99(1)^{b}$ & 6.4 & 0.312 (S) & 0.4 \\
\hline & Flucon & $2.0(\mathrm{~S})$ & $8.0(S)$ & 4 & $2.0(\mathrm{~S})$ & 1 & $8.0(S)$ & 4 \\
\hline & Itra & 0.25 (S-DD) & 0.25 (S-DD) & 1 & 0.25 (S-DD) & 1 & 0.25 (S-DD) & 1 \\
\hline & Micon & $0.39(\mathrm{~S})$ & $0.78(S)$ & 2 & $0.39(\mathrm{~S})$ & 1 & 0.546 (S) & 0.71 \\
\hline \multirow[t]{5}{*}{ C. albicans } & $A m B$ & $0.039(\mathrm{~S})$ & $0.0195(\mathrm{~S})$ & 0.5 & 0.0195 (S) & 0.5 & 0.039 (S) & 1 \\
\hline & $5-F C$ & $0.78(S)$ & $0.1(\mathrm{~S})$ & 0.1299 & $0.39(\mathrm{~S})$ & 0.5 & $0.078(\mathrm{~S})$ & 0.1 \\
\hline & Flucon & $0.125(\mathrm{~S})$ & 0.125 (S) & 1 & $0.125(\mathrm{~S})$ & 1 & $0.5(\mathrm{~S})$ & 4 \\
\hline & Itra & $0.03125(\mathrm{~S})$ & 0.0156 (S) & (2) & 0.03125 (S) & 1 & 0.0625 (S) & 2 \\
\hline & Micon & $0.39(\mathrm{~S})$ & 0.0245 (S) & 0.0629 & $0.78(S)$ & 2 & 0.01757 (S) & 0.045 \\
\hline \multirow[t]{5}{*}{ C. tropicalis } & AmB & $0.039(\mathrm{~S})$ & $0.0195(\mathrm{~S})$ & 0.5 & $0.039(\mathrm{~S})$ & 1 & $0.156(\mathrm{~S})$ & 4 \\
\hline & $5-\mathrm{FC}$ & $0.195(\mathrm{~S})$ & $0.0975(\mathrm{~S})$ & 0.5 & 4.99 (I) & 25.6 & $0.156(\mathrm{~S})$ & 0.8 \\
\hline & Flucon & $>128.0(\mathrm{R})$ & $>128.0(\mathrm{R})$ & $>1$ & $>128(\mathrm{R})$ & $>1$ & $>128$ (R) & $>1$ \\
\hline & Itra & $>64.0$ (R) & $>64.0(\mathrm{R})$ & $>1$ & $>64$ (R) & $>1$ & $>64$ (R) & $>1$ \\
\hline & Micon & $1.56(\mathrm{~S})$ & $0.78(S)$ & 0.5 & $1.56(\mathrm{~S})$ & 1 & $9.048(\mathrm{R})$ & 5.8 \\
\hline \multirow[t]{5}{*}{ C. neoformans } & $A m B$ & $0.039(\mathrm{~S})$ & $0.0195(\mathrm{~S})$ & 0.5 & $0.039(\mathrm{~S})$ & 1 & $0.039(\mathrm{~S})$ & 1 \\
\hline & $5-\mathrm{FC}$ & $6.25(1)^{b}$ & $6.25(I)$ & 1 & $0.78(\mathrm{~S})$ & 0.125 & $0.78(\mathrm{~S})$ & 0.125 \\
\hline & Flucon & $8.0(\mathrm{R})$ & $8.0(\mathrm{R})$ & 1 & $8.0(R)$ & 1 & $8.0(R)$ & 1 \\
\hline & Itra & 0.5 (S-DD) & 0.5 (S-DD) & 1 & 0.5 (S-DD) & 1 & 0.5 (S-DD) & 1 \\
\hline & Micon & 0.9766 (S) & 0.1953 (S) & 0.2 & 0.09766 (S) & 0.1 & 4.492 (S) & 4.6 \\
\hline \multirow[t]{5}{*}{ C. glabrata } & $A m B$ & $0.078(\mathrm{~S})$ & 0.0195 (S) & 0.25 & $0.039(\mathrm{~S})$ & 0.5 & $3.12(\mathrm{R})$ & 40 \\
\hline & $5-\mathrm{FC}$ & $8.0(\mathrm{I})$ & $4.0(I)$ & 0.5 & $51.2(\mathrm{R})$ & 6.4 & $1.6(I)$ & 0.2 \\
\hline & Flucon & 0.09766 (S) & 0.3906 (S) & 4 & $0.195(\mathrm{~S})$ & 2 & $0.195(\mathrm{~S})$ & 2 \\
\hline & Itra & $2.0(\mathrm{R})$ & $>64$ (R) & $>32$ & $>64$ (R) & $>32$ & $>64$ (R) & $>32$ \\
\hline & Micon & $0.39(\mathrm{~S})$ & $0.78(S)$ & 2 & 0.39 & 1 & $17.94(\mathrm{R})$ & 46 \\
\hline \multirow[t]{5}{*}{ C. krusei } & $\mathrm{AmB}$ & $0.078(\mathrm{~S})$ & 0.039 (S) & 0.5 & $0.078(\mathrm{~S})$ & 1 & 0.078 (S) & 1 \\
\hline & $5-\mathrm{FC}$ & $12.5(\mathrm{I})$ & $12.5(\mathrm{I})$ & 1 & $12.5(\mathrm{I})$ & 1 & $5.0(1)$ & 0.4 \\
\hline & Flucon & $64.0(\mathrm{R})$ & $64.0(\mathrm{R})$ & 1 & $64.0(\mathrm{R})$ & 1 & $64.0(\mathrm{R})$ & 1 \\
\hline & Itra & $1.0(\mathrm{R})$ & $1.0(\mathrm{R})$ & 1 & $1.0(\mathrm{R})$ & 1 & $1.0(R)$ & 1 \\
\hline & Micon & $0.39(\mathrm{~S})$ & $0.78(S)$ & 2 & $1.56(\mathrm{~S})$ & 4 & 8.97 (R) & 23 \\
\hline
\end{tabular}

amphotericin B (AmB), flucytosine (5-FC) fluconazole (Flucon), itraconazole (Itra) , and miconazole (Micon)

${ }^{\mathrm{b}} \mathrm{S}=$ sensitive; $\mathrm{R}=$ resistant; $\mathrm{I}=$ =intermediate resistance; $\mathrm{S}-\mathrm{DD}=$ sensitive based on reported disc diffusion concentration

${ }^{\mathrm{c}}$ Gluc=glucose; ${ }^{\mathrm{d}}$ Insu=insulin 
Table 2. Effect of insulin and glucose in RPMI medium with human plasma (50\% v/v; RPMI-P) on the MIC ( $\mu \mathrm{g} / \mathrm{ml})$

\begin{tabular}{|c|c|c|c|c|c|c|c|c|c|}
\hline Organism & Drug & RPMI-P ${ }^{c}$ & $\begin{array}{c}\text { Ratio } \\
\frac{\text { RPMI-P }}{\text { RPMI }}\end{array}$ & $\begin{array}{l}\text { RPMI-P \& } \\
\text { Glu }^{d}\end{array}$ & $\begin{array}{c}\begin{array}{c}\text { Ratio } \\
\text { RPMI-P \& }\end{array} \\
\begin{array}{c}\text { Gluc } \\
\text { RPMI-P }\end{array}\end{array}$ & $\begin{array}{l}\text { RPMI-P \& } \\
\text { Insu }^{\text {e }}\end{array}$ & $\begin{array}{c}\text { Ratio } \\
\text { RPMI-P \& Insu } \\
\text { RPMI-P }\end{array}$ & $\begin{array}{l}\text { RPMI-P, Gluc } \\
\quad \& \text { Insu }\end{array}$ & $\begin{array}{c}\text { Ratio } \\
\underline{\text { RPMI-P, }}, \underline{\text { Gluc }} \\
\underline{\text { \& Insu RPMI-P }}\end{array}$ \\
\hline \multirow[t]{5}{*}{ C. Iusitaniae } & $A m B^{a}$ & $0.078(S)^{b}$ & 2 & $9.75 \times 10^{-3}(S)$ & 0.25 & 0.0195 (S) & 0.25 & 0.0195 (S) & 0.25 \\
\hline & $5-\mathrm{FC}^{\mathrm{a}}$ & $0.78(\mathrm{~S})$ & 5 & $0.78(\mathrm{~S})$ & 1 & $0.78(\mathrm{~S})$ & 1 & $0.78(\mathrm{~S})$ & 1 \\
\hline & Fluco & $1.0(\mathrm{~S})$ & 0.25 & $1.0(\mathrm{~S})$ & 1 & $4.0(S)$ & 4 & $4.0(S)$ & 1 \\
\hline & $\operatorname{Itra}^{a}$ & $2.0(R)^{b}$ & 4 & $2.0(R)$ & 1 & $2.0(\mathrm{R})$ & 1 & $2.0(\mathrm{R})$ & 1 \\
\hline & Micon & $>36.075(\mathrm{R})$ & $>185$ & $>36.075(\mathrm{R})$ & $\geq 1$ & $0.3608(S)$ & 0.01 & $>36.08(R)$ & $\geq 1$ \\
\hline \multirow[t]{5}{*}{ C. parapsilosis } & $\mathrm{AmB}$ & 0.0195 (S) & 0.25 & $0.078(\mathrm{~S})$ & 4 & $0.039(\mathrm{~S})$ & 2 & 0.0195 (S) & 1 \\
\hline & $5-\mathrm{FC}$ & $24.96(I)^{b}$ & 32 & $24.96(I)$ & 1 & $24.96(I)$ & 1 & $24.96(I)$ & 1 \\
\hline & Fluco & $1.0(\mathrm{~S})$ & 0.5 & $0.5(\mathrm{~S})$ & 0.5 & $8.0(S)$ & 8 & $0.5(\mathrm{~S})$ & 0.5 \\
\hline & Itra & 0.125 (S) & 0.5 & 0.125 (S) & 1 & $0.125(S)$ & 1 & 0.5 (S-DD) & 2 \\
\hline & Micon & $>39.88$ (R) & $>92$ & $>39.88$ (R) & 1 & >39.88 (R) & $\geq 1$ & $>39.88$ (R) & $\geq 1$ \\
\hline \multirow[t]{5}{*}{ C. albicans } & $\mathrm{AmB}$ & 0.00975 (S) & 0.25 & 0.0195 (S) & 2 & 0.0195 (S) & 2 & 0.0195 (S) & 2 \\
\hline & $5-\mathrm{FC}$ & 3.12 (S) & 4 & 3.12 (S) & 1 & 3.12 (S) & 1 & 3.12 (S) & 1 \\
\hline & Fluco & $0.075(\mathrm{~S})$ & 0.5 & 0.075 (S) & 1 & $>6.8(\mathrm{~S}-\mathrm{R})$ & 1024 & $0.3(\mathrm{~S})$ & 4 \\
\hline & Itra & $0.03125(\mathrm{~S})$ & 1 & $0.03125(\mathrm{~S})$ & 1 & $0.03125(\mathrm{~S})$ & 1 & $0.03125(\mathrm{~S})$ & 1 \\
\hline & Micon & $0.39(\mathrm{~S})$ & 1 & $0.065(\mathrm{~S})$ & 0.166 & 0.0325 (S) & 0.083 & $0.78(\mathrm{~S})$ & 2 \\
\hline \multirow[t]{5}{*}{ C. tropicalis } & $\mathrm{AmB}$ & 0.0195 (S) & 0.5 & 0.0195 (S) & 1 & 0.0195 (S) & 1 & 0.0195 (S) & 1 \\
\hline & $5-\mathrm{FC}$ & $3.12(S)$ & 16 & $3.12(\mathrm{~S})$ & 1 & $3.12(\mathrm{~S})$ & 1 & 3.12 (S) & 1 \\
\hline & Fluco & $>128$ (R) & $>1$ & $>128(\mathrm{R})$ & 1 & $>128.0$ (R) & $>1$ & $>128.0$ (R) & 1 \\
\hline & Itra & $>64$ (R) & 1 & $>64$ (R) & 1 & $>64.0(\mathrm{R})$ & 1 & $>64.0(R)$ & 1 \\
\hline & Micon & $>35.88$ (R) & $>23$ & $>35.88$ (R) & 1 & $>35.88$ (R) & $\geq 1$ & $>35.88$ (R) & $\geq 1$ \\
\hline \multirow[t]{5}{*}{ C. neoformans } & $\mathrm{AmB}$ & $0.00488(\mathrm{~S})$ & 0.125 & 0.00975 (S) & 2 & 0.00975 (S) & 1 & 0.00975 (S) & 2 \\
\hline & $5-\mathrm{FC}$ & $25.0(\mathrm{I}-\mathrm{R})$ & 4 & $12.5(\mathrm{I})$ & 0.5 & $12.5(I)$ & 0.5 & $12.5(I)$ & 0.5 \\
\hline & Fluco & $1.0(\mathrm{~S})$ & 0.125 & $0.25(\mathrm{~S})$ & 0.25 & $1.0(\mathrm{~S})$ & 1 & $2.0(\mathrm{~S})$ & 2 \\
\hline & Itra & 0.25 (S- & 0.5 & 0.25 (S-DD) & 1 & 0.0125 (S) & 0.05 & 0.25 (S-DD) & 1 \\
\hline & Micon & 1.953 (S) & 2 & 0.3906 (S) & 0.2 & $0.078(\mathrm{~S})$ & 0.04 & $7.812(\mathrm{R})$ & 4 \\
\hline \multirow[t]{5}{*}{ C. glabrata } & $\mathrm{AmB}$ & $\begin{array}{l}3.71 \times 10^{-5} \\
\text { (S) }\end{array}$ & $4.76 \times 10^{-4}$ & $3.7 \times 10^{-5}(\mathrm{~S})$ & 1 & $3.71 \times 10^{-5}(S)$ & 1 & $3.71 \times 10^{-5}(\mathrm{~S})$ & 1 \\
\hline & $5-\mathrm{FC}$ & 0.0615 (S) & $7.69 \times 10^{-3}$ & $<0.0615$ (S) & $<1$ & $<0.0615$ (S) & $\leq 1$ & $0.246(S)$ & 4 \\
\hline & Fluco & $<2.4 \times 10^{-}$ & $<2.4 \times 10^{-4}$ & $<2.4 \times 10^{-5}(\mathrm{~S})$ & $<1$ & $<2.4 \times 10^{-5}(\mathrm{~S})$ & 1 & $<2.4 \times 10^{-5}(\mathrm{~S})$ & $\leq 1$ \\
\hline & Itra & 0.0194 (S) & $9.7 \times 10^{-3}$ & 0.0194 (S) & 1 & 0.0194 (S) & 1 & 0.0194 (S) & 1 \\
\hline & Micon & $1.08 \times 10^{-3}$ & $2.8 \times 10^{-3}$ & $5.41 \times 10^{-4}(S)$ & 0.5 & 0.0964 (S) & 89 & 0.001083 (S) & 1 \\
\hline \multirow[t]{5}{*}{ C. krusei } & $\mathrm{AmB}$ & 0.156 (S) & 2 & $0.624(\mathrm{~S})$ & 4 & $0.156(S)$ & 1 & $0.156(\mathrm{~S})$ & 1 \\
\hline & $5-\mathrm{FC}$ & $>25.0$ (I-R) & $>2$ & $>25.0(\mathrm{I}-\mathrm{R})$ & 1 & $>25.0(\mathrm{I}-\mathrm{R})$ & $\geq 1$ & $>25.0$ (I-R) & $\geq 1$ \\
\hline & Fluco & $64.0(R)$ & 1 & $64.0(R)$ & 1 & $64.0(\mathrm{R})$ & 1 & $64.0(\mathrm{R})$ & 1 \\
\hline & Itra & $4.0(\mathrm{R})$ & 4 & $4.0(\mathrm{R})$ & 1 & $4.0(\mathrm{R})$ & 1 & $4.0(\mathrm{R})$ & 1 \\
\hline & Micon & $35.88(R)$ & $>92$ & $8.97(R)$ & 0.25 & $574.08(R)$ & 16 & $>35.88$ (R) & $\geq 1$ \\
\hline
\end{tabular}

a amphotericin B (AmB), flucytosine (5-FC) fluconazole (Flucon), itraconazole (Itra), and miconazole (Micon)

${ }^{\mathrm{b}} \mathrm{S}=$ sensitive; R=resistant; I=intermediate resistance; $\mathrm{S}-\mathrm{DD}=$ sensitive based on reported disc diffusion concentration

${ }^{\mathrm{C}} \mathrm{P}=$ plasma

${ }^{\mathrm{d}}$ Gluc=glucose

e Insu=insulin

Thus, the addition of plasma to the medium resulted in an overall decrease in the concentration of miconazole, itraconazole, 5-fluorocytosine, and amphotericin B needed to inhibit the growth of $C$. glabrata (Table 3). In contrast, plasma overall caused an increase in the concentration of miconazole, itraconazole, 5-fluorocytosine, and amphotericin B needed to inhibit the growth of C. lusitaniae, C. tropicalis and C. krusei. The presence of the medium supplements insulin and/or glucose does not affect fungal MIC or MLC in a predictable manner regarding specific antifungal agents or organisms tested. The growth rate and relative production of yeast and/or hyphal forms in medium with and without glucose and/or insulin do not appear to be related to changes in the organism's MIC or MLC. 
Table 3. Ratio of effects of insulin and glucose on antifungal activity of amphotericin B, flucytosine, fluconazole, itraconazole, and miconazole in the presence of RPMI with $50 \%$ human plasma to homologous RPMI medium alone

\begin{tabular}{|c|c|c|c|c|c|}
\hline Organism & Drug & $\begin{array}{c}\begin{array}{c}\text { Ratio } \\
\text { RPMI-Plasma }\end{array} \\
\text { RPMI }\end{array}$ & $\begin{array}{c}\text { Ratio } \\
\frac{\text { RPMI-Pc } \& \text { Gluc }^{\mathrm{d}}}{\text { RPMI-Gluc }}\end{array}$ & $\begin{array}{c}\text { Ratio } \\
\frac{\text { RPMI-P \& Insu }}{\text { RPMI-Insu }}\end{array}$ & $\begin{array}{c}\text { Ratio } \\
\text { RPMI-P, Gluc \& Insu } \\
\text { RPMI-Gluc \& Insu }\end{array}$ \\
\hline \multirow[t]{5}{*}{ C. Iusitaniae } & $A m B$ & 2.0 & 0.5 & 1.0 & 0.25 \\
\hline & $5-\mathrm{FC}$ & 5.0 & 5.0 & 5.0 & 83.3 \\
\hline & Flucon & 0.25 & 0.125 & 2.0 & 0.25 \\
\hline & Itra & 4.0 & 0.5 & 4.0 & 32.0 \\
\hline & Micon & $>185.0$ & $>185.0$ & 1.85 & 16.1 \\
\hline \multirow[t]{5}{*}{ C. parapsilosis } & $A m B$ & 0.25 & 4.0 & 1.0 & 0.25 \\
\hline & $5-\mathrm{FC}$ & 32.0 & 64.0 & 5.0 & 80.0 \\
\hline & Flucon & 0.5 & 0.0625 & 4.0 & 0.0625 \\
\hline & Itra & 0.5 & 0.48 & 0.5 & 2.0 \\
\hline & Micon & $>92.0$ & $>51.13$ & $>102.3$ & $>73$ \\
\hline \multirow[t]{5}{*}{ C. albicans } & $\mathrm{AmB}$ & 0.25 & 1.0 & 1.0 & 0.5 \\
\hline & $5-\mathrm{FC}$ & 4.0 & 31.2 & 8.0 & 40 \\
\hline & Flucon & 0.5 & 0.6 & 54.4 & 0.6 \\
\hline & Itra & 1.0 & 2.0 & 1.0 & 0.5 \\
\hline & Micon & 1.0 & 2.65 & 0.042 & 44.4 \\
\hline \multirow[t]{5}{*}{ C. tropicalis } & $A m B$ & 0.5 & 1.0 & 0.5 & 0.125 \\
\hline & $5-\mathrm{FC}$ & 16.0 & 32.0 & 0.625 & 20.0 \\
\hline & Flucon & $>1.0$ & 1.0 & 1.0 & 1.0 \\
\hline & Itra & 1.0 & 1.0 & 1.0 & 1.0 \\
\hline & Micon & $>23.0$ & 46.0 & 23.0 & 3.97 \\
\hline \multirow[t]{5}{*}{ C. neoformans } & $\mathrm{AmB}$ & 0.125 & 0.5 & 0.25 & 0.25 \\
\hline & $5-\mathrm{FC}$ & 4.0 & 2.0 & 16.0 & 16.0 \\
\hline & Flucon & 0.125 & 0.03 & 0.125 & 0.25 \\
\hline & Itra & 0.5 & 0.5 & 0.025 & 0.5 \\
\hline & Micon & 2.0 & 2.0 & 0.7987 & 1.74 \\
\hline \multirow[t]{5}{*}{ C. glabrata } & $A m B$ & $4.76 \times 10^{-4}$ & $1.897 \times 10^{-3}$ & $9.5 \times 10^{-4}$ & $1.2 \times 10^{-5}$ \\
\hline & $5-\mathrm{FC}$ & $7.69 \times 10^{-3}$ & $1.5 \times 10^{-2}$ & $1.2 \times 10^{-3}$ & $1.54 \times 10^{-1}$ \\
\hline & Flucon & $<2.4 \times 10^{-4}$ & $6.1 \times 10^{-5}$ & $1.23 \times 10-4$ & $1.23 \times 10^{-4}$ \\
\hline & Itra & $9.7 \times 10^{-3}$ & $3 \times 10^{-4}$ & $3 \times 10^{-4}$ & $3 \times 10^{-4}$ \\
\hline & Micon & $2.8 \times 10^{-3}$ & $6.9 \times 10^{-4}$ & $2.47 \times 10^{-1}$ & $6.0 \times 10^{-5}$ \\
\hline \multirow[t]{5}{*}{ C. krusei } & $A m B$ & 2.0 & 16.0 & 2.0 & 2.0 \\
\hline & $5-\mathrm{FC}$ & $>2.0$ & 2.0 & 2.0 & 5.0 \\
\hline & Flucon & 1.0 & 1.0 & 1.0 & 1.0 \\
\hline & Itra & 4.0 & 4.0 & 4.0 & 4.0 \\
\hline & Micon & $>92.0$ & 11.5 & 368.0 & 4.0 \\
\hline
\end{tabular}

a amphotericin B (AmB), flucytosine (5-FC) fluconazole (Flucon), itraconazole (Itra), and miconazole (Micon)

${ }^{\mathrm{b}} \mathrm{S}=$ sensitive; $\mathrm{R}=$ resistant; $\mathrm{I=intermediate}$ resistance; $\mathrm{S}-\mathrm{DD}=$ sensitive based on reported disc diffusion concentration

${ }^{\mathrm{c}} \mathrm{P}=$ plasma; ${ }^{\mathrm{d}} \mathrm{Gluc}=$ glucose; ${ }^{\mathrm{e}}$ Insu=insulin

\section{Conclusions}

The testing of fungal susceptibility in the presence of plasma with and without the supplements glucose and/or insulin to simulate type II diabetes appears warranted in light of their sometimes adverse effect on the MIC of the antifungal agents. These results taken together indicate that alterations in the levels of serum components associated with type II diabetes, and simulated herein, cause an alteration in the in 
vitro activity of certain antifungal agents that is also dependent on the presence of plasma. Since treatment failure in candidal infections has been an issue and is increasing, it is likely that a portion of treatment efficacy failure is due to phenotypic resistance $[28,29]$. While multiple in vitro studies show that glucose induces phenotypic resistance, the organism is always exposed to glucose in the presence of insulin in vivo $[27,30,31]$. Most commonly, these studies focused on the effect glucose has on end-point determinations for amphotericin B, fluconazole and itraconazole [32]. This study demonstrated that testing of individual serum components, e.g., insulin and glucose in vitro showed that not only does insulin or glucose affect susceptibility in a manner that was species and drug-specific, but that the interaction of the two components can negate the effect, either positive or negative, of the other additive. This impact of insulin and glucose is further compounded by testing in the presence of human plasma vs. RPMI medium alone. These unpredictable phenotypic changes in susceptibility to antifungal agents were demonstrated for amphotericin B and C. glabrata, which were sensitive to the drug in medium alone or supplemented with either glucose or insulin. However, when combined, the MIC was $>40$-fold higher than medium alone or with either glucose or insulin. This effect was eliminated with the addition of anti-candidal antibodynegative plasma. In summary, these findings present evidence that a precision approach is needed for the determination of fungal drug susceptibility, particularly for individuals with type II diabetes.

Acknowledgements: The authors thank the Midwestern University Office of Research and Sponsored Programs and the Midwestern College of Graduate Studies for their support of this work.

Conflict of interest: The authors declare that no conflict of interest exists.

\section{References}

[1] A.M. Willis, W.A. Coulter, C.R. Fulton, J.R. Hayes, P.M. Bell, P.-J. Lamey. Oral candidal carriage and infection in insulin-treated diabetic patients. Diabetic Medicine 16 (1999) 675-679. https://doi.org/10.1046/i.1464-5491.1999.00134.x.

[2] H.M. Calvet, T.T. Yoshikawa. Infections in diabetes. Infectious Disease Clinics of North America 15 (2001) 407-421. https://doi.org/10.1016/s0891-5520(05)70153-7.

[3] M.A. de Resende, L.V.N.F. de Sousa, R.C.B.W. de Oliveira, C.Y. Koga-Ito, J.P. Lyon. Prevalence and Antifungal Susceptibility of Yeasts Obtained from the Oral Cavity of Elderly Individuals. Mycopathologia 162 (2006) 39-44. . https://doi.org/10.1007/s11046-006-0029-6

[4] R.H.P. Goncalves, E.T. Miranda, J.E. Zaia, M. Giannini. Species diversity of yeast in oral colonization of insulin-treated diabetes mellitus patients. Mycopathologia 162 (2006) 83-89. https://doi.org/10.1007/s11046-006-0038-5.

[5] M.F. Gonzalez-Lara, L. Ostrosky-Zeichner. Invasive Candidiasis. Seminars in Respiratory and Critical Care Medicine 41 (2020) 3-12. https://doi.org/10.1055/s-0040-1701215.

[6] O. Leroy, J.P. Gangneux, P. Montravers, J.P. Mira, F. Gouin, J.P. Sollet, J. Carlet, J. Reynes, M. Rosenheim, B. Regnier, O. Lortholary, G. AmarCand Study Group. Epidemiology, management, and risk factors for death of invasive Candida infections in critical care: A multicenter, prospective, observational study in France (2005-2006). Critical Care Medicine 37 (2009) 1612-1618. https://doi.org/10.1097/ccm.0b013e31819efac0.

[7] T.S. Ng, M.N.M. Desa, D. Sandai, P.P. Chong, L.T.L. Than. Growth, biofilm formation, antifungal susceptibility and oxidative stress resistance of Candida glabrata are affected by different glucose concentrations. Infection, Genetics and Evolution 40 (2016) 331-338. https://doi.org/10.1016/j.meegid.2015.09.004.

[8] M.H. Nguyen, C.J. Clancy, V.L. Yu, Y.C. Yu, A.J. Morris, D.R. Snydman, D.A. Sutton, M.G. Rinaldi. Do in vitro susceptibility data predict the microbiologic response to amphotericin $B$ ? Results of a 
prospective study of patients with Candida fungemia. Journal of Infectious Diseases 177 (1998) 425430. https://doi.org/10.1086/514193.

[9] A. Rodaki, I.M. Bohovych, B. Enjalbert, T. Young, F.C. Odds, N.A. Gow, A.J. Brown. Glucose promotes stress resistance in the fungal pathogen Candida albicans. Molecular biology of the cell 20 (2009) 4845-4855. https://doi.org/10.1091/mbc.e09-01-0002.

[10] A. Becker, R. Roth. Insulysin and pitrilysin: insulin-degrading enzymes of mammals and bacteria. Methods Enzymol. 248 (1995) 693-703. https://doi.org/10.1016/0076-6879/95)48046-3.

[11] S. Christensen. Insulin rescues the unicellular eucaryote Tetrahymena from dying in a complete, synthetic nutrient medium. Cell Biol. Internat. 17 (1993) 833-837. https://doi.org/10.1006/cbir.1993.1145.

[12] S.E. Fawell, J. Lenard. A specific insulin receptor and tyrosine kinase activity in the membranes of Neurospora crassa. Biochemical and Biophysical Research Communications 155 (1988) 59-65. https://doi.org/10.1016/s0006-291x(88)81049-0.

[13] D. LeRoith, M. Lesniak, J. Roth. Insulin in insects and annelids. Diabetes 30 (1981) 70-76. https://doi.org/10.2337/diab.30.1.70.

[14] D. LeRoith, J. Shiloach, R. Heffron, C. Rubinovitz, R. Tanenbaum, J. Roth. Insulin-related material in microbes: similarities and differences from mammalian insulins. Can. J. Biochem. Cell Biol. 63 (1985) 839-849. https://doi.org/10.1139/085-106.

[15] S. Nolting, H. Hagemeier, K. Fegeler. Effect of insulin on germ tube and mycelial formation of Candida albicans. Mykosen 25 (1982).

[16] B.J. Plotkin, S.M. Viselli. Effect of insulin on microbial growth. Curr. Microbiol. 41 (2000) 60-64.

[17] N. Dabrowa, S. Taxer, D.H. Howard. Germination of Candida albicans induced by proline. Infection and immunity 13 (1976) 830-835. https://doi.org/10.1007/s002840010092.

[18] E. Evans, F. Odds, M. Richardson, K. Holland. Optimum conditions for initiation of filamentation in Candida albicans. Canadian Journal of Microbiology 21 (1975) 338-342. https://doi.org/10.$1139 / \mathrm{m} 75-048$.

[19] A. Man, C.N. Ciurea, D. Pasaroiu, A.-I. Savin, F. Toma, F. Sular, L. Santacroce, A. Mare. New perspectives on the nutritional factors influencing growth rate of Candida albicans in diabetics. An in vitro study. Memórias do Instituto Oswaldo Cruz 112 (2017) 587-592. https://doi.org/10.1590/0074$\underline{02760170098 .}$.

[20] N.C.f.C.L. Standards., Reference method for broth dilution antifungal susceptibility testing of yeasts; tentative standard, National Committee for Clinical Laboratory Standards, Wayne, Pa, 1995.

[21] J.L. Rodriguez-Tudela, J. Berenguer, J.V. Martinez-Suarez, R. Sanchez. Comparison of a spectrophotometric microdilution method with RPMI-2\% glucose with the National Committee for Clinical Laboratory Standards reference macrodilution method M27-P for in vitro susceptibility testing of amphotericin B, flucytosine, and fluconazole against Candida albicans. Antimicrobial Agents and Chemotherapy 40 (1996) 1998-2003. https://doi.org/10.1128/aac.40.9.1998.

[22] O.T. Malazy, M. Shariat, R. Heshmat, F. Majlesi, M. Alimohammadian, N.K. Tabari, B. Larijani. Vulvovaginal candidiasis and its related factors in diabetic women. Taiwanese Journal of Obstetrics and Gynecology 46 (2007) 399-404. https://doi.org/10.1016/s1028-4559(08)60010-8.

[23] C. Amar, J. Ashish, V. Hajare, B. Yogesh. Study of prevalence and antifungal susceptibility of candida. International Journal of Pharma and Bio Sciences 4 (2013). https://doi.org/10.1186/s12879-019$\underline{4736-2 .}$.

[24] K.-H. Lin, C.-M. Chen, T.-L. Chen, S.-C. Kuo, C.-C. Kao, Y.-C. Jeng, M.-W. Ho. Diabetes mellitus is associated with acquisition and increased mortality in HIV-uninfected patients with cryptococcosis: a population-based study. Journal of Infection 72 (2016) 608-614. https://doi.org/10.1016/i.jinf.2016.$\underline{01.016 .}$ 
[25] R. Acharya, K. Khanal, P. Upadhyaya, S. Kafle, V. Savaliya. Diabetes mellitus as a risk factor for cryptococcal meningitis in immunocompetent. IDCases 22 (2020) e00988. https://doi.org/10.1016/j.idcr.2020.e00988.

[26] R. Diamond, J. Bennett. Prognostic factors in cryptococcal meningitis: a study in 111 cases. Annals of internal medicine 80 (1974) 176-181. https://doi.org/10.7326/0003-4819-80-2-176.

[27] I.V. Ene, A.K. Adya, S. Wehmeier, A.C. Brand, D.M. MacCallum, N.A. Gow, A.J. Brown. Host carbon sources modulate cell wall architecture, drug resistance and virulence in a fungal pathogen. Cellular microbiology 14 (2012) 1319-1335. https://doi.org/10.1111/i.1462-5822.2012.01813.x.

[28] J.D. Sobel. Treatment of vaginal Candida infections. Expert Opinion on Pharmacotherapy 3 (2002) 1059-1065. https://doi.org/10.1517/14656566.3.8.1059.

[29] N.B. McClenny, H. Fei, E.J. Baron, A.C. Gales, A. Houston, R.J. Hollis, M.A. Pfaller. Change in colony morphology of Candida lusitaniae in association with development of amphotericin B resistance. Antimicrobial Agents and Chemotherapy 46 (2002) 1325-1328. https://doi.org/10.1128/aac.46.5.1325-1328.2002.

[30] S.M. Mandal, D. Mahata, L. Migliolo, A. Parekh, P.S. Addy, M. Mandal, A. Basak. Glucose directly promotes antifungal resistance in the fungal pathogen, Candida spp. Journal of Biological Chemistry 289 (2014) 25468-25473. https://doi.org/10.1074/jbc.c114.571778.

[31] L. Knight, J. Fletcher. Growth of Candida albicans in saliva: stimulation by glucose associated with antibiotics, corticosteroids, and diabetes mellitus. The Journal of infectious diseases 123 (1971) 371377. https://doi.org/10.1093/infdis/123.4.371.

[32] M.H. Nguyen, Y.Y. Christine. Influence of incubation time, inoculum size, and glucose concentrations on spectrophotometric endpoint determinations for amphotericin B, fluconazole, and itraconazole. Journal of clinical microbiology 37 (1999) 141-145. https://doi.org/10.1128/jcm.37.1.141-145.1999. 